\title{
On the kinetic study of electrochemical vapour deposition
}

\author{
L.G.J. de Haart, Y.S. Lin, K.J. de Vries and A.J. Burggraaf \\ Laboratory of Inorganic Chemistry, Materials Science and Catalysis, Faculty of Chemical Technology, University of Twente, \\ P.O. Box 217,7500 AE Enschede, The Netherlands
}

Received 12 June 1991; accepted for publication 19 June 1991

\begin{abstract}
A theoretical analysis is presented which quantitatively describes the transition behaviour of the kinetics of the electrochemical vapour deposition of yttria-stabilized zirconia on porous substrates. It is shown that up to a certain deposition time and corresponding film thickness the rate limiting step is oxygen diffusion through the substrate pores, giving a linear dependence of the film thickness on the deposition time. For longer deposition times, i.e. thicker films, a transition of the rate limiting step to bulk electrochemical diffusion in the film occurs, resulting in a parabolic dependence of the film thickness on the deposition time. Simulation results are presented to show the effects of the experimental conditions on this transition time.
\end{abstract}

\section{Introduction}

Electrochemical Vapour Deposition (EVD) has in recent years become a powerful technique in the production of thin gas tight yttria-stabilized zirconia (YSZ) electrolyte layers on porous substrates for use in Solid Oxide Fuel Cells (SOFC) [1-6]. The principles of the process have been reported previously [1-3,7]. In short, the porous substrate separates a mixture of metal chloride $\left(\mathrm{ZrCl}_{4}\right.$ and $\left.\mathrm{YCl}_{3}\right)$ vapours in a carrier gas stream (usually $\mathrm{Ar}$ ) from an oxygen source reactant (e.g. a mixture of water vapour and hydrogen or air). In the initial CVD stage of the process the reactants from both sides of the substrate inter-diffuse in the substrate pores and react to form the solid oxide which is deposited on the substrate pore wall. Usually the experimental conditions are so chosen that deposition mainly takes place near the entrance of the substrate pore from which side the metal chloride vapours enter. Prolonged deposition causes the pores to become plugged with the solid oxide, thus separating the metal chloride vapours from the oxygen source reactant. In the subsequent EVD stage of the process water or oxygen is reduced at the oxygen/solid oxide interface inside the pore. The oxygen ions migrate through the (mixed conducting) solid oxide to the metal chloride/solid interface. Here the oxygen ions react with the metal chloride vapours to form again the solid oxide, thus causing continued growth of the plugs on the metal chloride vapour side. Eventually this will lead to an EVD solid oxide film on top of the substrate.

In several studies the kinetics of the EVD stage have been modelled [4,5,8-10]. In most of these studies only the electrochemical transport through the growing EVD film was considered as rate-limiting step. This yields a parabolic dependence of the EVD film thickness $H$ on the deposition time. This parabolic behaviour was experimentally observed by Carolan and Michaels [8] and Pal and Singhal [4,9].

In a previous paper [10] we introduced a model which took into account all major mass transport steps involved in the EVD stage: (1) oxygen/water diffusion through the substrate pores to the oxygen/ film interface; (2) oxygen/water reduction at the oxygen/film interface; (3) bulk electrochemical transport in the growing film; and (4) oxidation reaction at the metal chloride/film interface. It was shown that under the experimental conditions of a small substrate pore size to pore-length ratio the first step of oxygen/water diffusion through the substrate pores would be rate limiting. This yields a linear dependence of the EVD film thickness $H$ on the deposition time. This linear behaviour was experimentally observed for this type of substrates for short deposition times [11]. Furthermore, it was shown 
that a parabolic relation between the film thickness and the deposition time should be observed when the electrochemical transport through the EVD film is rate limiting. The transition of the rate limiting step from pore diffusion to bulk diffusion limitation was, however, not studied in this previous work [10].

In this paper we present an improved analysis of the kinetics of the EVD process, which quantitatively explains both the linear and parabolic behaviour. The effects of the process parameters, like the substrate microstructure (pore radius and length) and experimental conditions (total and oxygen partial pressures and temperature) on the transition behaviour are discussed using the results of the theoretical analysis.

\section{Model}

In the previous paper [10] it has been shown, that both charge transfer steps (step 2 reduction and step 4 oxidation) are most likely not rate limiting in the EVD process. Therefore both steps are neglected here to simplify the analysis. The total oxygen partial pressure drop over the system is $P_{\mathrm{O}(\mathrm{w})}-P_{\mathrm{O}(\mathrm{m})}$, where $P_{\mathrm{O}(w)}$ is the oxygen partial pressure in the oxygen source reactant outside the substrate and $P_{\mathrm{O}(\mathrm{m})}$ the oxygen partial pressure in the metal chlorides vapour mixture. The total oxygen partial pressure drop is divided over the two remaining mass transport steps considered: across the substrate pores to the oxygen/film interface $P_{\mathrm{O}(\mathrm{w})}-P_{\mathrm{O}(1)}$ and across the EVD film $P_{\mathrm{O}(\mathrm{i})}-P_{\mathrm{O}(\mathrm{m})}$, where $P_{\mathrm{O}(\mathrm{i})}$ is the oxygen partial pressure inside the substrate pores at the oxygen/film interface. The oxygen flux $J$ (in $\frac{1}{2} \mathrm{O}_{2}$ ) is now given by:

for the diffusion of oxygen in the substrate pore [10]:

$J=\frac{2 D_{\mathrm{O}}}{L R T}\left(P_{\mathrm{O}(\mathrm{w})}-P_{\mathrm{O}(\mathrm{i})}\right)$

for the electrochemical diffusion of oxygen in the EVD film [10]:

$$
\begin{aligned}
J= & \frac{1}{H} \frac{R T}{2 F^{2}}\left\{\sigma_{\mathrm{h}}^{0}\left(\left[P_{\mathrm{O}(\mathrm{i})}\right]^{1 / 4}-\left[P_{\mathrm{O}(\mathrm{m})}\right]^{1 / 4}\right)\right. \\
& \left.+\sigma_{n}^{\mathrm{O}}\left(\left[P_{\mathrm{O}(\mathrm{m})}\right]^{-1 / 4}-\left[P_{\mathrm{O}(\mathrm{i})}\right]^{-1 / 4}\right)\right\} .
\end{aligned}
$$

The growth rate of the EVD film is given by the mass balance:

$\frac{\mathrm{d} H}{\mathrm{~d} t}=\frac{V_{\mathrm{m}} J}{2}$.

In eq. (1) $D_{0}$ is the effective diffusivity for oxygen in the substrate pore calculated from the Knudsen diffusivity $D_{\mathrm{K}}$ according to:

$$
\begin{aligned}
D_{\mathrm{O}} & =\frac{\epsilon}{\tau} D_{\mathrm{K}} \\
& =\frac{\epsilon}{\tau} \frac{2}{3} r\left(\frac{8 R T}{\pi M_{\mathrm{O}}}\right)^{1 / 2} .
\end{aligned}
$$

The other symbols used in eqs. (1)-(4) are explained in table 1 .

In eqs. (1)-(3) all parameters are known except $J, P_{O(i)}$ and $H$, the EVD film thickness. To find the functions of $J, P_{\mathrm{O}(\mathrm{i})}$ and $H$ with respect to the deposition time $t$ all three equations have to be solved simultaneously. This set of nonlinear algebraic and differential equations is first transformed to a set of dimensionless initial value differential equations using the method reported by Audry-Sanchez [12]. Since the oxygen is supplied by substrate pore diffusion, this step must initially be rate limiting when the EVD film is smaller than a certain value (referred to later on as a transition film thickness $H_{\mathrm{T}}$ ). The transformed eqs. (1)-(3) can be solved with the following initial conditions to give the functions $J(t), H(t)$ and $P_{O(i)}(t)$ :

$$
\text { at } t=0 \text { : }
$$$$
\left\{\begin{array}{l}
P_{\mathrm{O}(\mathrm{i})}=P_{\mathrm{O}(\mathrm{m})} \\
H=0 \\
J=J_{\max }=\frac{2 D_{\mathrm{O}}}{L R T}\left(P_{\mathrm{O}(\mathrm{w})}-P_{\mathrm{O}(\mathrm{m})}\right) .
\end{array}\right.
$$

When $P_{O(w)} \gg P_{O(m)}$, eq. $(5 c)$ reduces to:

$$
J_{\max }=\frac{2 D_{\mathrm{O}} P_{\mathrm{O}(w)}}{L R T} .
$$


Table 1

List of symbols and values for parameters used in eqs. (1)-(4).

\begin{tabular}{|c|c|c|c|}
\hline Symbol & Description & Value & Unit \\
\hline$\epsilon$ & porosity of the substrate & 0.5 & \\
\hline$\tau$ & tortuosity of the substrate & 2 & \\
\hline$r$ & mean pore radius of the substrate & $1.00 \times 10^{-7}$ & $\mathrm{~m}$ \\
\hline$L$ & thickness of substrate & $2.0 \times 10^{-3}$ & $\mathrm{~m}$ \\
\hline$V_{M}$ & average molar volume of YSZ & $2.07 \times 10^{-5}$ & $\mathrm{~m}^{3} \mathrm{~mol}^{-1}$ \\
\hline$M_{\mathrm{O}}$ & molar weight of oxygen & 0.032 & $\mathrm{~kg} \mathrm{~mol}^{-1}$ \\
\hline$P_{\mathrm{w}}$ & partial water vapor pressure in water chamber & 100 & $\mathrm{~Pa}$ \\
\hline$R$ & gas constant & 8.314 & $\mathrm{~J} \mathrm{~mol}^{-1} \mathrm{~K}^{-1}$ \\
\hline$F$ & Faraday constant & 96487 & $\mathrm{C} \mathrm{mol}^{-1}$ \\
\hline$T$ & absolute temperature & 1273 & $\mathrm{~K}$ \\
\hline$D_{0}$ & effective oxygen diffusivity & $1.53 \times 10^{-5}$ & $\mathrm{~m}^{2} \mathrm{~s}^{-1}$ \\
\hline$\sigma_{\mathrm{h}}^{0}$ & hole conductivity of YSZ at $1273 \mathrm{~K}$ and $P_{\mathrm{O}_{2}}=10^{5} \mathrm{~Pa}$ & $3.25 \times 10^{-4 a)}$ & $\mathrm{S} \mathrm{m}^{-1} \mathrm{~Pa}^{-1 / 4}$ \\
\hline$\sigma_{n}^{0}$ & electron conductivity of YSZ at $1273 \mathrm{~K}$ and $P_{\mathrm{O}_{2}}=10^{5} \mathrm{~Pa}$ & $1.03 \times 10^{-5 a)}$ & $\mathrm{S} \mathrm{m}^{-1} \mathrm{~Pa}^{1 / 4}$ \\
\hline$P_{\mathrm{O}(\mathrm{w})}$ & oxygen partial pressure in water chamber & 20 & $\mathrm{~Pa}$ \\
\hline$P_{\mathrm{O}(\mathrm{m})}$ & oxygen partial pressure in metal chloride chamber & $2 \times 10^{-7}$ & $\mathrm{~Pa}$ \\
\hline$P_{\mathrm{O}(\mathrm{i})}$ & oxygen partial pressure at water/film interface & variable & $\mathrm{Pa}$ \\
\hline$H$ & EVD film thickness & variable & $\mathrm{m}$ \\
\hline$J$ & oxygen flux (for $\mathrm{O}^{2-}$ ) & variable & $\mathrm{mol} \mathrm{m}{ }^{-2} \mathrm{~s}^{-1}$ \\
\hline
\end{tabular}

a) Value obtained from ref. [13] for $8 \mathrm{~m} / \mathrm{o} \mathrm{YSZ}$.

\section{Numerical analysis}

The standard parameter values used in the calculations are given in table 1 . The results of the calctlation are given in fig. 1. Fig. I A shows the oxygen flux $J$ relative to $J_{\max }$ as function of the deposition time. It shows initially a constant (the maximum) oxygen flux for a certain period, which corresponds to the period in which the pore diffusion is rate limiting. In this period the EVD film thickness $H$ is a linear function of the deposition time as is shown in fig. 1C. After a certain time the EVD film has increased to a thickness which causes the oxygen flux through the film (eq. (2)) to become dominant over the pore diffusion flux. The total oxygen flux starts to decrease after this time (see fig. 1A) indicating the transition from pore diffusion control to bulk electrochemical diffusion control. At the same time the EVD film thickness starts to deviate from the linear behaviour and becomes parabolic (see fig. 1C).

Since the curve of the relative oxygen flux versus deposition time shows the most pronounced change, we define the transition time $t_{\mathrm{T}}$ as the time at which the relative oxygen flux equals 0.95 . The thickness the EVD film has reached at this time $t_{\mathrm{T}}$ is defined as the transition thickness $H_{\mathrm{T}}$. Both $t_{\mathrm{T}}$ and $H_{\mathrm{T}}$ are

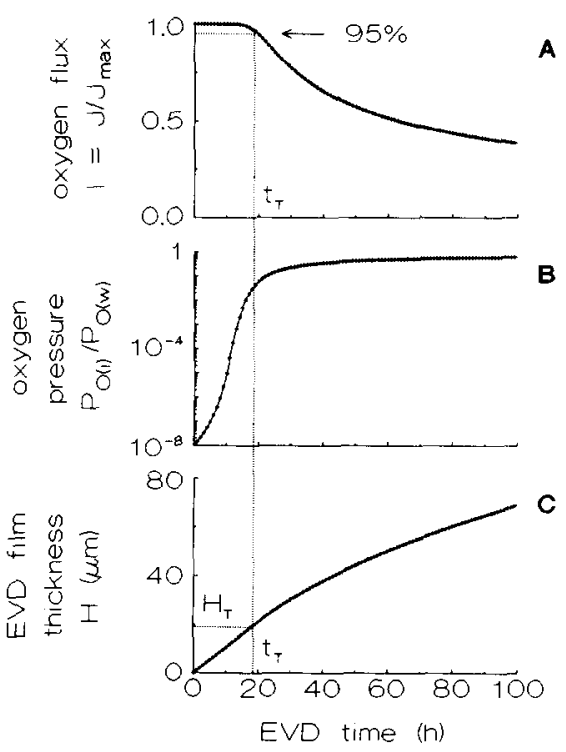

Fig. 1. Calculation results of the EVD kinetics at standard conditions ( see table 1). As function of the deposition time: (A) the oxygen flux $I=J / J_{\max }$; (B) the oxygen partial pressure at the oxygen $/$ film interface $Y_{\mathrm{i}}=P_{\mathrm{O}(\mathrm{i})} / P_{\mathrm{O}(w)}$; (C) the EVD film thickness $H$. Indicated are the transition time $t_{\mathrm{T}}$ and thickness $H_{\mathrm{T}}$. 
indicated in fig. 1 . For the results shown in fig. 1 the transition time is around $19 \mathrm{~h}$ which corresponds to a transition thickness of around $21 \mu \mathrm{m}$. The linear film growth rate constant $k_{1}$ in this initial period can be easily obtained from:

$k_{1}=H_{\mathrm{T}} / t_{\mathrm{I}}$

and amounts in this example $1.1 \mu \mathrm{m} / \mathrm{h}$.

Fig. 1B shows the path of the oxygen partial pressure at the oxygen/film interface $P_{O(i)}$ (relative to $\left.P_{O(w)}\right)$ during the EVD film growth process. Starting from the value of $P_{\mathrm{O}(\mathrm{m})}$ it increases gradually with deposition time, around $t_{\mathrm{T}}$ steeply, until it approaches the value of $P_{O(w)}$.

The next step in the analysis is to correlate the thus defined transition time $t_{\mathrm{T}}$ and transition thickness $H_{\mathrm{T}}$ to the experimental conditions of the EVD process. In fig. 2 the results are shown of the calculations of both $t_{\mathrm{T}}$ and $H_{\mathrm{T}}$ as function of the parameter $\beta$ defined as $2 D_{\mathrm{O}} P_{\mathrm{O}(\mathrm{w})} / L$. This parameter $\beta$ thus comprises all characteristics of the substrate: the porosity $\epsilon$, the tortuosity $\tau$, the average pore radius $r$ ( see eq. $(4) ; D_{O}$ ) and the thickness of the substrate $L$, as well as the oxygen partial pressure in the water chamber.

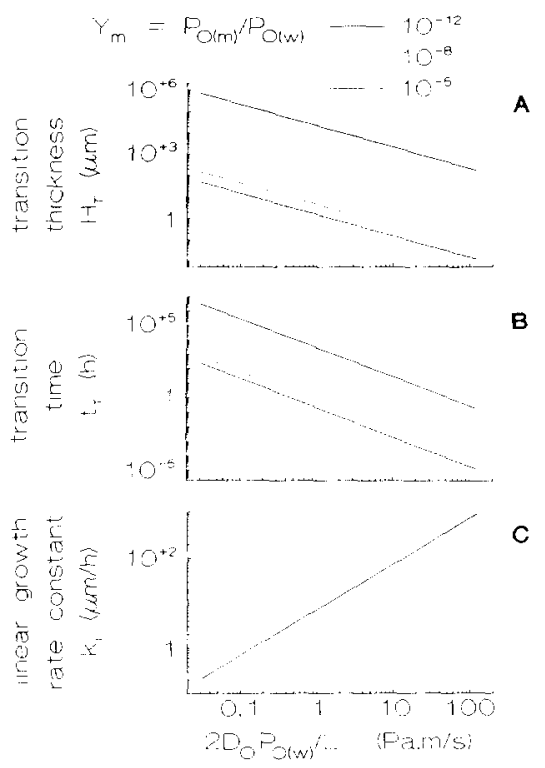

Fig. 2. Effect of the substrate characteristics $\left(\beta=2 D_{\mathrm{O}} P_{\mathrm{O}(w)} / L\right)$ on: (A) the transition thickness $H_{\mathrm{T}}$; (B) the transition time $t_{\mathrm{T}}$; (C) the linear growth rate constant $k_{\mathrm{l}}$. Values for all other parameters are standard.
Fig. 2 shows that with increasing $\beta$ both the transition time and the transition thickness decrease. This can be easily understood, since when the maximum pore diffusion flux increases, the time on which the EVD film has reached the thickness such that the bulk diffusion will become dominant will decrease. Regression results show that $H_{\mathrm{T}}$ is proportional to $1 / \beta$ and $t_{\mathrm{T}}$ to $1 / \beta^{2}$, indicating the large effect of the substrate characteristics on the transition behaviour and thus on the rate limiting step in the process. For a substrate with a large pore radius to substrate thickness ratio $(r / L)$ the transition time is very short, corresponding to a very short period in which the film thickness will increase linearly with deposition time.

Shown in fig. 2 is also the effect of the oxygen partial pressure in the chloride chamber $P_{O(m)}$ (relative to $\left.P_{\mathrm{O}(w)}\right)$ on the transition thickness and time. It is clear that the smaller this ratio is (i.e. the larger the oxygen partial pressure difference) the longer (thicker) the transition time (thickness) is.

In fig. $2 \mathrm{C}$ the linear film growth rate constant $k_{1}$ is also shown as function of $\beta$. From the dependences of both $H_{\mathrm{T}}$ and $t_{\mathrm{T}}$ on $\beta$ it follows directly that $k_{1}$ is proportional to $\beta$. This is in line with eq. $(5 \mathrm{c})$. Fig. 2C also shows that $k_{1}$ is independent on $P_{\mathrm{O}(\mathrm{m})}$, at least for the values used in the calculations. This is in line with eq. (6), since in all these cases $P_{\mathrm{O}(\mathrm{w})} \gg P_{\mathrm{O}(\mathrm{m})}$. The fact that the linear growth rate constant in the case of pore diffusion limitation is independent on $P_{\mathrm{O}(\mathrm{m})}$ (for $P_{\mathrm{O}(\mathrm{m})}$ two or more orders of magnitude smaller than $P_{\mathrm{O}(\mathrm{w})}$ ) was already pointed out in the previous study [10].

In fig. 3 the effect of the deposition temperature on the transition thickness and time is shown, again with different ratios of $Y_{\mathrm{m}}=P_{\mathrm{O}(\mathrm{m})} / P_{\mathrm{O}(\mathrm{w})}$. A higher deposition temperature causes an increase in transition time (and thickness). This can be understood by examining the temperature dependence of eq. (1) (combined with eq. (4)) and of eq. (2). The pore diffusion flux is proportional to $T^{-1 / 2}$. The maximum oxygen flux (eq. (6)) in the system will thus decrease with increasing temperature. The bulk diffusion flux in the film is proportional to $T$ times the sum of the exponential temperature dependences of the partial hole and electron conductivities of the oxide (see eq. (2)). Since the latter two increase with increasing temperature, the bulk diffusion flux will increase considerably too. This means that at a higher 


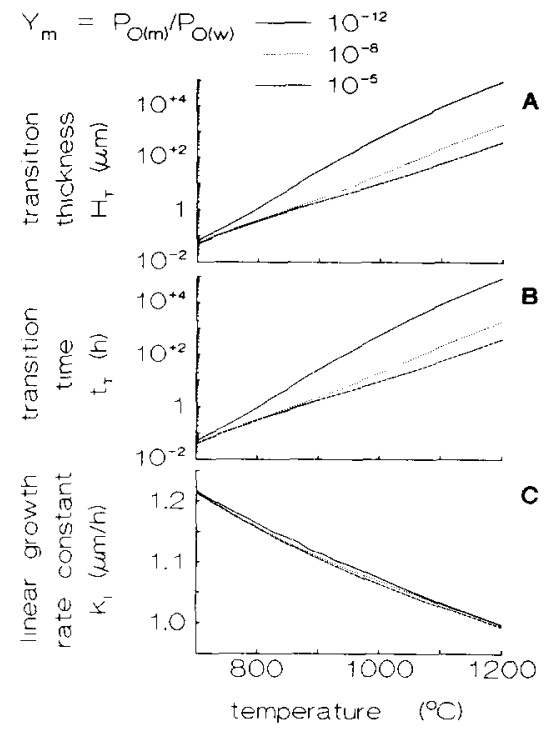

Fig. 3. Effect of the deposition temperature on: (A) the transition thickness $H_{\mathrm{T}}$; (B) the transition time $t_{\mathrm{T}}$; (C) the linear growth rate constant $k_{1}$. Values for all other parameters are standard.

temperature the film thickness has to increase considerably for the oxygen flux in the film to be constant. This requires a longer deposition time. Moreover, because of the decrease in the maximum pore diffusion flux, i.e. a slower growth rate, the deposition time must be even so longer. In other words, the transition time will increase with increasing temperature.

Shown in fig. 3C is the temperature dependence of the linear film growth rate constant. Again as could be expected there is no influence of $P_{\mathrm{O}(\mathrm{m})}$. Regression analysis of the data shows $k_{1}$ to be proportional to $T^{-1 / 2}$, which is in line with eq. (6). Comparing figs. $2 \mathrm{C}$ and $3 \mathrm{C}$ it can be seen that the influence of the deposition temperature on the linear growth rate constant is less significant than the influence of the substrate characteristics. A change of the substrate pore diameter to pore length ratio can cause the linear growth rate constant to change over several orders of magnitude, whereas a change in deposition temperature has only a minor effect.

The calculation results thus show, that the EVD process is governed by at least two mass transport steps: (1) oxygen diffusion through the substrate pores and (2) electrochemical oxygen diffusion through the growing film. Initially the first step is rate limiting, untill the growing film has reached the thickness at which step 2 becomes dominant. It is shown that the transition time is mostly affected by the substrate characteristics. In case of a substrate with a small pore-radius to pore-length ratio this time is of the order of several hours to days. Increasing the poreradius to pore-length ratio decreases this time to several minutes or seconds. In practical situations deposition experiments are performed with deposition times of several hours. The experiments performed by de Haart et al. [11] on a substrate with a small pore-radius to pore-length ratio $(\beta=0.15)$ indeed show a linear dependence of the EVD film thickness on deposition time for the first five hours. For longer deposition times a deviation from this linear behaviour appears. Pal and Singhal [4,9] use a substrate with a much larger substrate pore-radius to porelength ratio $(\beta \approx 15)$. The transition time in this case will be of the order of several seconds to minutes. The experimental deposition times are however of the order of several tens of minutes to several hours. This causes that the linear region is not observed experimentally by these authors and they come to the justified conclusion that the bulk electrochemical oxygen diffusion in the growing film is the rate limiting step.

\section{Conclusion}

The improved analysis on the kinetics of the EVD process quantitatively describes the transition from substrate pore diffusion limitation in the initial period of the process to bulk electrochemical diffusion limitation. In this initial period the film growth is linear in time. After the transition it becomes parabolic. The substrate characteristics (pore-radius to pore-length ratio ) and experimental conditions have a large influence on the transition time (and thickness). Experimental conditions (deposition time) can mask the presence of the initial period.

\section{Acknowledgements}

The investigations were partly supported by the Dutch Ministry of Economical Affairs (Research 
Project "ECVD Synthesis and Properties of Porous Composite Systems", IOP Technical Ceramics No. $87 \mathrm{~A} 045$ ) and partly performed under contract with the Commission of the European Communities (CEC) within the Non-Nuclear Energy $R$ and $D$ Programme (Research Contract "Membrane Based Thin Layer SOFC Technology", No. EN3E-0175NL).

\section{References}

[1] A.O. Isenberg, in: Electrode Materials and Processes for Energy Conversion and Storage, Proc. Symp., Philadelphia, PA, 1977, eds. J.D.E. McIntyre, S. Srinivasan and F.G. Will, Proc. Vol. 77-6 (The Electrochem. Soc. Inc., Princeton NJ, 1977) p. 572.

[2] G. Dietrich and W. Schäfer, Int. J. Hydrogen Energy 9 (1984) 747.

[3] M.F. Carolan and J.N. Michaels, Solid State Ionics 25 (1987) 207.

[4] U.B. Pal and S.C. Singhal, in: Solid Oxide Fuel Cells, Proc. First Intern. Symp., Hollywood, FL, 1989, ed. S.C. Singhal, Proc. Vol. 89-11, (The Electrochem. Soc. Inc., Pennington NJ, 1989) p. 41.
[5] J.P. Dekker, N.J. Kiwiet and J. Schoonman, in: Solid Oxide Fuel Cells, Proc. First Intern. Symp., Hollywood, FL, 1989 , ed. S.C. Singhal, Proc. Vol. 89-11 (The Electrochem. Soc. Inc., Pennington NJ, 1989) p. 57.

[6] Y.S. Lin, L.G.J. de Haart, K.J. de Vries and A.J. Burggraaf, in: Solid Oxide Fuel Cells, Proc. First Intern. Symp., Hollywood, FL, 1989, ed. S.C. Singhal, Proc. Vol. 89-11, (The Electrochem. Soc. Inc., Pennington NJ, 1989) p. 67.

[7] Y.S. Lin, L.G.J. de Haart, K.J. de Vries and A.J. Burggraaf, in: Euro-ceramics, Vol. 3, Engineering Ceramics, Proc. First Europ. Ceram. Soc. Conf., Maastricht, 1989, eds. G. de With, R.A. Terpstra and R. Metselaar (Elsevier, Amsterdam, 1989) p. 3590.

[8] M.F. Carolan and J.N. Michaels. Solid State Ionics 37 (1990) 189.

[9] U.B. Pal and S.C. Singhal, J. Electrochem. Soc. 137 (1990) 2937.

[10] Y.S. Lin, L.G.J. de Haart, K.J. de Vries and A.J. Burggraaf, J. Electrochem. Soc. 137 (1990) 3960.

[11] L.G.J. de Haart, Y.S. Lin, K.J. de Vries and A.J. Burggraaf, J. Europ. Ceram. Soc. 8 (1991) 59.

[12] J. Audry-Sanchez, Can. J. Chem. Eng. 66 (1988) 1031.

[13] J.H. Park and R.N. Blumenthal, J. Electrochem. Soc. 136 (1989) 2867. 